\title{
MALPIGHIACEAE DE COLOMBIA: PATRONES DE DISTRIBUCIÓN, RIQUEZA, ENDEMISMO Y DIVERSIDAD FILOGENÉTICA
}

\author{
Diego Giraldo-Cañas
}

Herbario Nacional Colombiano (COL), Instituto de Ciencias Naturales, Universidad Nacional de Colombia, Bogotá D. C., Colombia; dagiraldoc@unal.edu.co (autor corresponsal).

\begin{abstract}
Giraldo-Cañas, D. 2021. Malpighiaceae from Colombia: Patterns of distribution, richness, endemism, and phylogenetic diversity. Darwiniana, nueva serie 9(1): 39-54.
\end{abstract}

Malpighiaceae constitutes a family of 77 genera and ca. 1300 species, distributed in tropical and subtropical regions of both hemispheres. They are mainly diversified in the American continent and distributed in a wide range of habitats and altitudinal gradients. For this reason, this family can be a model plant group to ecological and biogeographical analyses, as well as evolutive studies. In this context, an analysis of distribution, richness, endemism and phylogenetic diversity of Malpighiaceae in natural regions and their altitudinal gradients was undertaken. Malpighiaceae are represented in Colombia by 34 genera and 246 species (19.1\% of endemism). Thus, Colombia and Brazil (44 genera, 584 species, $61 \%$ of endemism) are the two richest countries on species of this family. The highest species richness and endemism in Colombia is found in the lowlands (0-500 m a.s.1.: 212 species, 28 endemics); only ten species are distributed on highlands (2500-3200 m a.s.1.). Of the Malpighiaceae species in Colombia, Heteropterys leona and Stigmaphyllon bannisterioides have a disjunct amphi-Atlantic distribution, and six other species show intra-American disjunctions. Both richness and endemism decrease with altitude $\left(y=-0.061 x+173.57 ; \mathrm{R}^{2}=0.82 ; y=-0.009 x+27.76 ; \mathrm{R}^{2}=0.95\right.$, respectively). Amazonia (116 species, 4 endemics) and the Andes (89 species, 23 endemics) exhibit the highest richness among the family. In Colombia, 15 of the 19 clades among the family are represented, where the most diversified are the Stigmaphyllon clade (5 genera, 48 species, 10 endemics), the Byrsonima clade (3/39/5) and the Hiraea clade (3/31/9). The relationship of phylogenetic diversity with altitude is similar to the pattern of specific richness by altitudinal interval. Amazonia, Orinoquia, and Magdalena Valley show highest phylogenetic diversity. These results, combined with those of other highly diversified biological groups in the country, could be important to define and delimitate new priority areas for conservation in Colombia.

Keywords. Altitudinal gradient; Amazonia; Andes; Malpighiales; Neotropical flora.

Resumen. Giraldo-Cañas, D. 2021. Malpighiaceae de Colombia: Patrones de distribución, riqueza, endemismo y diversidad filogenética. Darwiniana, nueva serie 9(1): 39-54.

Las Malpighiaceae constituyen una familia de 77 géneros y ca. 1300 especies, distribuidas en regiones tropicales y subtropicales de ambos hemisferios. Éstas están principalmente diversificadas en el continente americano y se distribuyen en una amplia variedad de hábitats y gradientes altitudinales. Por tal motivo, pueden constituir un grupo vegetal modelo a la hora de emprender análisis ecológicos, biogeográficos y evolutivos. En este contexto, se analizó la distribución, la riqueza, el endemismo y la diversidad filogenética de la familia en las regiones naturales y del gradiente altitudinal en Colombia. Las Malpighiaceae están representadas en Colombia por 34 géneros y 246 especies (19,1\% de endemismo); así, Colombia y Brasil (44 géneros, 584 especies, $61 \%$ de endemismo) son los dos países más ricos a nivel mundial. La mayor riqueza de especies y el mayor endemismo, se da en las tierras bajas (0-500 m s.m.: 212 especies, 28 endémicas). Sólo diez especies se distribuyen en las tierras altas (2500-3200 m s.m.). De las especies presentes en Colombia, Heteropterys leona y Stigmaphyllon bannisterioides exhiben una distribución disyunta anfiatlántica, $\mathrm{y}$ otras seis especies muestran disyunciones intra-americanas. La riqueza y el endemismo disminuyen con la altitud $\left(y=-0,061 x+173,57 ; \mathrm{R}^{2}=0,82 ; y=-0,009 x+27,76 ; \mathrm{R}^{2}=0,95\right.$, respectivamente). 
La Amazonia (116 especies, 4 endémicas) y los Andes ( 89 especies, 23 endémicas) exhiben la riqueza más alta. En Colombia están representados 15 de los 19 clados reconocidos para la familia, siendo los más diversificados, el clado Stigmaphyllon (5 géneros, 48 especies, 10 endémicas), el clado Byrsonima $(3 / 39 / 5)$ y el clado Hiraea (3/31/9). La diversidad filogenética en cuanto a los gradientes altitudinales es similar al patrón de la riqueza específica por intervalo altitudinal. La Amazonia, la Orinoquia y el valle del río Magdalena son las regiones que muestran la mayor diversidad filogenética. Esta información, combinada con la de otros grupos biológicos ricamente expresados en el país, podría constituir un insumo para definir y delimitar nuevas áreas prioritarias para la conservación en Colombia.

Palabras claves. Amazonia; Andes; Flora neotropical; Gradiente altitudinal; Malpighiales.

\section{INTRODUCCIÓN}

Las revisiones taxonómicas y las diferentes fuentes de información biológica (catálogos, inventarios, bases de datos), son esenciales y constituyen los insumos básicos para entender los patrones biogeográficos y la organización espacial de las comunidades vegetales (Funk \& Richardson, 2002; Funk, 2006; Borges et al., 2010). En este sentido, la búsqueda de distintos tipos de patrones es un tema central y recurrente en biogeografía y ecología (Ferro \& Barquez, 2014). La visión tradicional de una disminución lineal del número de especies con la altitud fue interpretada alguna vez como un patrón universal, semejante al observado en los gradientes latitudinales (Ferro \& Barquez, 2014). Sin embargo, estudios recientes muestran que no existe un único patrón altitudinal de riqueza de especies, y que contrariamente al supuesto de una disminución monotónica en función de la altitud, el caso más común es un pico de riqueza en altitudes intermedias (Ferro \& Barquez, 2014), referido como el "efecto del dominio medio" (Colwell \& Lees, 2000; Adams, 2009; Giraldo-Cañas, 2014).

En este contexto, el conocimiento detallado de la variación altitudinal de la riqueza de especies en diferentes regiones del mundo es un requisito fundamental a la hora de indagar los procesos que producen dichos patrones (Colwell \& Lees, 2000; Grytnes \& Vetaas, 2002; Adams, 2009; Borges et al., 2010; Ferro \& Barquez, 2014). Por lo tanto, los gradientes altitudinales proveen un importante escenario para estudiar las relaciones de la distribución de especies y el clima, debido a la gran variación climática en pequeñas distancias geográficas (Lloret \& González-Mancebo, 2011; Giraldo-Cañas, 2014). En este sentido y dadas la riqueza y la amplia expresión ambiental de sus especies, la familia
Malpighiaceae Juss. sería un modelo para estudiar procesos de distribución en el neotrópico.

Las Malpighiaceae constituyen una familia de 77 géneros y ca. 1300 especies (Almeida et al., 2016; Davis et al., 2020a), distribuidas en regiones tropicales y subtropicales de ambos hemisferios, con cerca del 90\% de las especies presentes en el Nuevo Mundo (desde Arizona, Nuevo México, Texas y Florida en los Estados Unidos de América, el Caribe hasta la Argentina y Chile) y el restante $10 \%$, se encuentra en el Viejo Mundo (África, Australia, China, Filipinas, India, Indonesia, Madagascar, Malasia, Micronesia, Nepal, Nueva Caledonia, Nueva Guinea, Pakistán, Península Arábiga, Sri Lanka, Taiwán, Tailandia y varias islas del Pacífico) (Anderson et al., 2020). $\mathrm{Al}$ respecto, las Malpighiaceae se dispersaron desde Sudamérica vía el Caribe hacia Norteamérica y de ahí a Eurasia por el Atlántico norte y subsecuentemente, alcanzaron diferentes áreas tropicales del Viejo Mundo, cuando las condiciones paleoambientales así lo facilitaron (Davis \& Anderson, 2010).

La familia Malpighiaceae se caracteriza por variados hábitos de crecimiento, desde árboles, arbustos, sufrútices y muy raramente hierbas perennes en Aspicarpa Rich. (Anderson, 2016), hasta especies trepadoras, tanto leñosas como herbáceas, las cuales se distribuyen en una amplia gama de hábitats. Cabe destacar que las Malpighiaceae están entre las familias con más diversidad de especies trepadoras en el neotrópico, junto con las Apocynaceae, las Asteraceae, las Bignoniaceae, las Fabaceae, las Passifloraceae y las Sapindaceae, entre otras (Linares, 2001; Prósperi et al., 2001; Acevedo-Rodríguez, 2005; Abadía Bonilla et al., 2015). En este contexto, el objetivo de este trabajo es documentar los patrones de variación altitudinal en la riqueza, el endemismo y la diversidad filogenética de las Malpighiaceae en Colombia. 


\section{MATERIALES Y MÉTODOS}

Lacircunscripcióny la nomenclatura de los géneros y las especies está basada en Anderson (2006; 2013), Anderson \& Davis (2007; 2013), Davis \& Anderson (2010), Anderson \& Anderson (2016), Anderson et al. (2020), Davis et al. (2020b) y Almeida \& van den Berg (2021). Los datos de la representatividad de la familia Malpighiaceae en Colombia provienen de muestreos personales, de la revisión de las colecciones depositadas en los herbarios ACAM, AFP, ANDES, AS, BA, BAF, BRG, CAUP, CDMB, CEN, CEPEC, COAH, COL, CR, CUVC, CHOCO, EAN, F, FAUC, FMB, G, HECASA, HFAB, HORI, HPUJ, HUA, HUC, HUQ, IAN, IBGE, INPA, IPA, JAUM, LLANOS, MEDEL, MO, NY, PSO, QCA, RSA, SI, SURCO, TOLI, UDBC, UIS, UPTC, US y VALLE (Thiers, 2020), así como de Cuatrecasas (1958), Giraldo-Cañas $(1996 ; 2011)$ y Anderson \& Anderson (2016). A los ejemplares de herbario se les tomó toda la información de sus respectivas fichas, tales como departamento, municipio, localidad, altitud, coordenadas (aunque en la mayoría de los ejemplares de herbario este dato no figuraba), usos, nombres populares, estado reproductivo, fechas de colección, recolectores, números de colección y herbario. A la localidad de los ejemplares analizados se la clasificó por unidad biogeográfica en el contexto colombiano. Las unidades biogeográficas colombianas están basadas en Bernal et al. (2016). La consideración de las especies nuevas está basada en Anderson \& Anderson (2016). Por su parte, los términos riqueza/diversidad y endemismo están basados en Badii et al. (2007) y Noguera-Urbano (2017), respectivamente. La diversidad filogenética se basa en los postulados de Llopis-Belenguer et al. (2018) y Hernández-Ruedas et al. (2019) y aquí se hace una mirada desde la representatividad de los clados de la familia en Colombia. La configuración y los nombres de los clados de las Malpighiaceae está basada en Anderson et al. (2020). Se utilizaron intervalos altitudinales con una amplitud de 500 $\mathrm{m}$, amplitud frecuentemente utilizada en estudios de gradientes altitudinales (e.g. Stevens, 1992; Lægaard, 1999; Etter \& van Wyngaarden, 2000; Grytnes \& Vetaas, 2002; Kessler, 2002; Oommen \& Shanker, 2005; Giraldo-Cañas, 2014; 2018), lo cual facilita las comparaciones de riqueza, diversidad y endemismo entre diferentes grupos biológicos.
Adicionalmente, con el fin de identificar el patrón de variación con la altitud para la riqueza de especies y el endemismo, se usan los números de riqueza o endemismo por cada intervalo altitudinal, a partir de un análisis de regresión lineal simple (Daniel, 1990).

\section{RESULTADOS Y DISCUSIÓN}

Riqueza, endemismo y distribución. Las Malpighiaceae están representadas en Colombia por 34 géneros y 246 especies (Tablas 1 y 2); así, en Colombia está representado el 19\% de las especies de la familia a nivel mundial y el $44,2 \%$ de los géneros. Cabe destacar que Cuatrecasas (1958) halló para Colombia 21 géneros y 168 especies, así, en los 63 años entre ese trabajo y el actual, se ha incrementado en 78 el número de especies de Malpighiaceae para la flora de Colombia. El endemismo corresponde a 47 especies, lo cual constituye el 19,1\% de las especies presentes en Colombia. Así, Colombia es uno de los países más ricos en esta familia junto con Brasil (584 especies), Venezuela (189), México (169) y Perú (137) (Mamede et al., 2010; Villaseñor, 2016; Ulloa Ulloa et al., 2017; Almeida et al., 2021). Por su parte, los géneros más diversificados en Colombia son Byrsonima Kunth (36 especies), Heteropterys Kunth (28), Hiraea Jacq. (28), Stigmaphyllon A. Juss. (28), Tetrapterys Cav. (25), Bunchosia Kunth (23) y Mascagnia (DC.) Bertero (15). Este patrón de riqueza por género se ajusta, en líneas generales, a los patrones encontrados en otros países con numerosas Malpighiaceae (Mamede et al., 2010; Villaseñor, 2016; Ulloa Ulloa et al., 2017). En cuanto al hábito, las formas lianescentes suman 162 especies, los árboles y los arbustos representan 83 especies, y sólo hay en Colombia una especie correspondiente a un sufrútice, Byrsonima verbascifolia (Tabla 2).

La mayor riqueza de especies se da en las tierras bajas; así, en el primer intervalo altitudinal (0-500 m s.m.) se han encontrado 212 especies, lo cual representa el $86,2 \%$ de las especies presentes en Colombia, seguido del segundo intervalo (500-1000 m s.m.) con 94 especies $(38,2 \%)$ (Tabla 2), mientras que el límite altitudinal de las Malpighiaceae en Colombia (3200 m s.m.) sólo lo alcanzan dos especies, Stigmaphyllon bogotense, en los Andes y la Sierra Nevada de Santa Marta, y Stigmaphyllon columbicum, en los Andes. 
Tabla 1. Las Malpighiaceae de Colombia y su distribución por regiones biogeográficas y gradientes altitudinales. *: Especie endémica de Colombia.

Especie

Adelphia hiraea (Gaertn.) W.R. Anderson

Adelphia platyrachis (Triana \& Planch.) W.R. Anderson*

Alicia macrodisca (Triana \& Planch) Griseb.

Amorimia concinna (C.V. Morton) W.R. Anderson*

Andersoniodoxa marcelae (W.R. Anderson) C. Davis \&

Amorim*

Andersoniodoxa spruceana (Niedenzu) C. Davis \&

Amorim

Banisteriopsis elegans (Triana \& Planch.) Sandwith

Banisteriopsis martiniana (A. Juss.) Cuatrec.

Banisteriopsis muricata (Cav.) Cuatrec.

Banisteriopsis padifolia (Nied.) B. Gates

Banisteriopsis pubescens (Nied.) Cuatrec.

Banisteriopsis wilburii B. Gates

Blepharandra angustifolia (Kunth) W.R. Anderson

Blepharandra heteropetala W.R. Anderson

Bronwenia acapulcensis (Rose) W.R. Anderson \& C. Davis

Bronwenia cornifolia (Kunth) W.R. Anderson \& C. Davis

Bronwenia wurdackii (B. Gates) W.R. Anderson \& C. Davis

Bunchosia anomala W.R. Anderson*

Bunchosia argentea (Jacq.) DC.

Bunchosia armeniaca (Cav.) DC.

Bunchosia brevistyla W.R. Anderson*

Bunchosia brevisurcularis Dobson

Bunchosia cauliflora W.R. Anderson

Bunchosia cestrifolia Cuatrec.

Bunchosia decussiflora W.R. Anderson

Bunchosia diphylla (Jacq.) Cuatrec. \& Croat

Bunchosia dwyeri Cuatrec. \& Croat

Bunchosia glandulifera (Jacq.) Kunth

Bunchosia hartwegiana Benth.*

Bunchosia macrophylla Rose

Bunchosia nitida (Jacq.) Rich.

Bunchosia odorata (Jacq.) Juss.

Bunchosia petraea W.R. Anderson

Bunchosia pseudonitida Cuatrec.

Bunchosia retusa Triana \& Planch.*

Bunchosia swartziana Griseb.

Bunchosia systyla (Nied.) Dobson

Bunchosia sp. nov. 1*
Regiones biogeográficas

Caribe, Pacífica, valle del río Magdalena

Pacífica

Amazonia, Andes, Guayana, Orinoquia

Caribe

Pacífica

Amazonia

Amazonia, Andes, Pacífica, Sierra Nevada de Santa Marta, valle del río Magdalena

Gradiente altitudinal (m s.m.)

0-1000

$0-500$

$0-1000$

$0-500$

$0-500$

$0-500$

Amazonia

Andes, Caribe, Orinoquia, valle del río Cauca, valle del río Magdalena

Andes, Pacífica

Andes

Andes, Pacífica, valle del río Cauca

$0-2000$

0-500

$0-2000$

$0-2500$

$1000-2000$

$0-2500$

$0-500$

$0-500$

Amazonia, Guayana

$0-500$

0-3000

Andes, Caribe, Pacífica, valle del río Magdalena

$0-1000$

Amazonia, Andes, Guayana, Orinoquia, valle del río

Cauca, valle del río Magdalena

$0-500$

Caribe, valle del río Cauca, valle del río Magdalena

Amazonia, Andes, Caribe, Pacífica, Sierra Nevada de Santa Marta, valle del río Cauca

$0-2500$

Amazonia, Andes, Orinoquia, Pacífica, valle del río

Cauca, valle del río Magdalena

$0-3000$

Andes

$1500-2000$

Caribe, Pacífica

$0-500$

$0-500$

$500-3000$

Andes, valle del río Magdalena

$0-500$

Caribe, Sierra Nevada de Santa Marta, valle del río

$0-1000$

Magdalena

Andes

$1000-1500$

Amazonia, Andes, Caribe, Guayana, Orinoquia, valle del río Magdalena

$0-2500$

Caribe, Sierra Nevada de Santa Marta, valle del río

Magdalena

Pacífica

$0-1000$

$0-500$

Andes, Caribe, Orinoquia, Pacífica, valle del río

Cauca, valle del río Magdalena

$0-2000$

Caribe

$0-500$

Guayana, Orinoquia

$0-500$

Andes, islas caribeñas, valle del río Cauca, valle del río Magdalena

$0-2000$

Andes

1000-1500

Islas caribeñas

$0-500$

Amazonia

$0-500$

Valle del río Cauca

500-1000


Tabla 1. (Continuación.).

\begin{tabular}{|c|c|c|}
\hline Especie & Regiones biogeográficas & $\begin{array}{l}\text { Gradiente } \\
\text { altitudinal } \\
\text { (m s.m.) }\end{array}$ \\
\hline Bunchosia sp. nov. 2 & Andes & $1000-3000$ \\
\hline Bunchosia sp. nov. 3 & Andes & $1500-2500$ \\
\hline Burdachia prismatocarpa A. Juss. & Amazonia, Guayana, Orinoquia & $0-500$ \\
\hline Burdachia sphaerocarpa A. Juss. & Amazonia, Guayana & $0-500$ \\
\hline Byrsonima aerugo Sagot & Andes, Pacífica & $0-1500$ \\
\hline Byrsonima amoena Cuatrec. & Amazonia, Guayana & $0-1000$ \\
\hline Byrsonima arthropoda A. Juss. & $\begin{array}{l}\text { Amazonia, Andes, Guayana, Orinoquia, Pacífico, } \\
\text { valle del río Cauca, valle del río Magdalena }\end{array}$ & $0-1500$ \\
\hline Byrsonima bronweniana W.R. Anderson & Amazonia, Guayana & $0-500$ \\
\hline Byrsonima bucidifolia Standl. & Islas caribeñas & $0-500$ \\
\hline Byrsonima chrysophylla Kunth & Amazonia, Orinoquia & $0-500$ \\
\hline Byrsonima concinna Benth. & Amazonia, Guayana & $0-500$ \\
\hline Byrsonima coniophylla A.Juss. & Amazonia, Guayana & $0-500$ \\
\hline Byrsonima crassifolia (L.) Kunth & $\begin{array}{l}\text { Amazonia, Andes, Caribe, islas caribeñas, Guayana, } \\
\text { Orinoquia, Pacífica, Sierra Nevada de Santa Marta, } \\
\text { valle del río Cauca, valle del río Magdalena }\end{array}$ & $0-2500$ \\
\hline Byrsonima crispa A. Juss. & $\begin{array}{l}\text { Amazonia, Andes, Guayana, Orinoquia, Pacífica, } \\
\text { valle del río Cauca, valle del río Magdalena }\end{array}$ & $0-1500$ \\
\hline Byrsonima cuprea Griseb. & Amazonia, Guayana & $0-500$ \\
\hline Byrsonima fernandezii Cuatrec. & Amazonia, Guayana & $0-500$ \\
\hline Byrsonima flexipes W.R. Anderson & Amazonia, Guayana & $0-500$ \\
\hline Byrsonima frondosa A. Juss. & Guayana & $0-500$ \\
\hline Byrsonima garcibarrigae Cuatrec. & $\begin{array}{l}\text { Amazonia, Andes, Pacífica, valle del río Cauca, valle } \\
\text { del río Magdalena }\end{array}$ & $0-2000$ \\
\hline Byrsonima hypoleuca Turcz. & Andes, Pacífica & $0-2000$ \\
\hline Byrsonima japurensis A. Juss. & Amazonia, Guayana, Orinoquia & $0-500$ \\
\hline Byrsonima krukoffii W.R.Anderson & Amazonia, Guayana & $0-500$ \\
\hline Byrsonima laevis Nied. & Amazonia, Guayana & $0-500$ \\
\hline Byrsonima linguifera Cuatrec. & Amazonia, Guayana, Orinoquia & $0-500$ \\
\hline Byrsonima luetzelburgii Steyerm. & Amazonia, Guayana & $0-500$ \\
\hline Byrsonima nemoralis Cuatrec.* & Andes, Pacífica & $0-500$ \\
\hline Byrsonima nitidissima Kunth & Guayana, Orinoquia & $0-500$ \\
\hline Byrsonima oblongifolia A. Juss. & Orinoquia & $0-500$ \\
\hline Byrsonima poeppigiana A. Juss. & Amazonia & $0-500$ \\
\hline Byrsonima punctulata A. Juss. & Amazonia, Guayana, Orinoquia & $0-500$ \\
\hline Byrsonima putumayensis Cuatrec. & Andes & $1000-1500$ \\
\hline Byrsonima schultesiana Cuatrec. & Amazonia, Guayana, Orinoquia & $0-500$ \\
\hline Byrsonima spicata (Cav.) Kunth & $\begin{array}{l}\text { Amazonia, Andes, Caribe, Guayana, Orinoquia, } \\
\text { Pacífica, Sierra Nevada de Santa Marta, valle del río } \\
\text { Cauca, valle del río Magdalena }\end{array}$ & $0-1500$ \\
\hline Byrsonima verbascifolia (L.) DC. & Amazonia, Andes, Caribe, Guayana, Orinoquia & $0-2000$ \\
\hline Byrsonima wurdackii W.R. Anderson & Amazonia, Guayana & $0-500$ \\
\hline Byrsonima sp. nov. 1 & Amazonia, Pacífica & $0-500$ \\
\hline Byrsonima sp. nov. $2^{*}$ & Andes & $1000-2500$ \\
\hline Byrsonima sp. nov. $3 *$ & Orinoquia & $0-500$ \\
\hline Byrsonima sp. nov. 4* & Andes & $1500-2500$ \\
\hline Byrsonima sp. nov. 5* & Sierra Nevada de Santa Marta & $1000-1500$ \\
\hline Callaeum antifebrile (Griseb.) D.M. Johnson & Amazonia & $0-500$ \\
\hline Carolus sinemariensis (Aubl.) W.R. Anderson & $\begin{array}{l}\text { Andes, Caribe, Guayana, Orinoquia, Pacífica, valle } \\
\text { del río Cauca, valle del río Magdalena }\end{array}$ & $0-2500$ \\
\hline
\end{tabular}


Tabla 1. (Continuación.).

\begin{tabular}{|c|c|c|}
\hline Especie & Regiones biogeográficas & $\begin{array}{l}\text { Gradiente } \\
\text { altitudinal } \\
\text { (m s.m.) }\end{array}$ \\
\hline Christianella glandulifera (Cuatrec.) W.R. Anderson & Amazonia & $0-500$ \\
\hline $\begin{array}{l}\text { Christianella mesoamericana (W.R. Anderson) W.R. } \\
\text { Anderson }\end{array}$ & Pacífica & $0-500$ \\
\hline Diacidia galphimioides Griseb. & Amazonia, Guayana & $0-1000$ \\
\hline Dicella julianii (J. F. Macbr.) W.R. Anderson & Amazonia, valle del río Cauca & $0-1000$ \\
\hline Diplopterys cabrerana (Cuatrec.) B.Gates & Amazonia, Andes & $0-1000$ \\
\hline Diplopterys cristata (Griseb.) W.R. Anderson \& C. Davis & Caribe, Sierra Nevada de Santa Marta & $0-500$ \\
\hline $\begin{array}{l}\text { Diplopterys erianthera (A. Juss.) W.R. Anderson \& C. } \\
\text { Davis }\end{array}$ & Amazonia & $0-500$ \\
\hline $\begin{array}{l}\text { Diplopterys heterostyla (A. Juss.) W.R. Anderson \& C. } \\
\text { Davis* }\end{array}$ & Caribe, valle del río Magdalena & $0-1000$ \\
\hline Diplopterys krukoffii (B. Gates) W.R. Anderson \& C. Davis & Amazonia, Guayana & $0-500$ \\
\hline Diplopterys longialata (Nied.) W.R. Anderson \& C. Davis & Amazonia & $0-500$ \\
\hline Diplopterys lucida (Rich.) W.R. Anderson \& C. Davis & $\begin{array}{l}\text { Andes, Sierra Nevada de Santa Marta, valle del río } \\
\text { Magdalena }\end{array}$ & $0-2000$ \\
\hline Diplopterys nutans (Nied.) W.R. Anderson \& C. Davis & Amazonia & $0-500$ \\
\hline Diplopterys platyptera (Griseb.) W.R. Anderson \& C. Davis & Caribe & $0-500$ \\
\hline $\begin{array}{l}\text { Diplopterys pubipetala (A. Juss.) W.R. Anderson \& C. } \\
\text { Davis }\end{array}$ & $\begin{array}{l}\text { Amazonia, Andes, Guayana, Orinoquia, Pacífica, } \\
\text { valle del río Magdalena }\end{array}$ & $0-1500$ \\
\hline Ectopopterys soejartoi W.R. Anderson & Valle del río Cauca, valle del río Magdalena & $0-1000$ \\
\hline Gaudichaudia albida Schltdl. \& Cham. & Andes & $1500-2500$ \\
\hline Glandonia williamsii Steyerm. & Amazonia, Guayana & $0-500$ \\
\hline Glicophyllum gracile (W.R. Anderson) R.F. Almeida & Amazonia, Guayana & $0-500$ \\
\hline Glicophyllum stylopterum (A. Juss.) R.F. Almeida & $\begin{array}{l}\text { Amazonia, Caribe, Orinoquia, Pacífica, valle del río } \\
\text { Cauca, valle del río Magdalena }\end{array}$ & $0-1500$ \\
\hline Heteropterys actinoctenia W.R. Anderson & Amazonia & $0-500$ \\
\hline Heteropterys alata (W.R. Anderson) W.R. Anderson & $\begin{array}{l}\text { Amazonia, Andes, Guayana, Orinoquia, valle del río } \\
\text { Magdalena }\end{array}$ & $0-1000$ \\
\hline Heteropterys atabapensis W.R. Anderson & Amazonia, Guayana & $0-500$ \\
\hline Heteropterys aureosericea Cuatrec. & Amazonia & $0-500$ \\
\hline Heteropterys ayacuchensis W.R. Anderson & Orinoquia & $0-500$ \\
\hline Heteropterys berteroana A. Juss. & Caribe & $0-500$ \\
\hline Heteropterys colombiana W.R. Anderson* & Caribe, valle del río Magdalena & $0-1000$ \\
\hline $\begin{array}{l}\text { Heteropterys complicata (Kunth) W.R. Anderson \& C. } \\
\text { Davis }\end{array}$ & Caribe, Orinoquia & $0-500$ \\
\hline Heteropterys floridana Cuatrec. & $\begin{array}{l}\text { Amazonia, Guayana, Orinoquia, valle del río Cauca, } \\
\text { valle del río Magdalena }\end{array}$ & $0-1000$ \\
\hline Heteropterys laurifolia (L.) A. Juss. & $\begin{array}{l}\text { Andes, Caribe, Sierra Nevada de Santa Marta, valle } \\
\text { del río Magdalena }\end{array}$ & $0-1500$ \\
\hline Heteropterys leona (Cav.) Exell & Pacífica & $0-500$ \\
\hline Heteropterys lonicerifolia Triana \& Planch. & Andes, Caribe, Sierra Nevada de Santa Marta & $0-2000$ \\
\hline Heteropterys macradena (DC.) W.R. Anderson & $\begin{array}{l}\text { Amazonia, Andes, Caribe, Guayana, Orinoquia, } \\
\text { Pacíica, Sierra Nevada de Santa Marta, valle del río } \\
\text { Magdalena }\end{array}$ & $0-1000$ \\
\hline Heteropterys macrostachya A. Juss. & $\begin{array}{l}\text { Amazonia, Andes, Caribe, Guayana, Orinoquia, } \\
\text { Sierra Nevada de Santa Marta, valle del río Cauca, } \\
\text { valle del río Magdalena }\end{array}$ & $0-2000$ \\
\hline Heteropterys magnifica W.R. Anderson & Amazonia & $0-500$ \\
\hline Heteropterys nervosa A. Juss. & Amazonia, Guayana & $0-1000$ \\
\hline Heteropterys oblongifolia Gleason & Amazonia, Guayana & $0-500$ \\
\hline Heteropterys obovata (Small) Cuatrec. \& Croat & Pacífica & $0-500$ \\
\hline
\end{tabular}


Tabla 1. (Continuación.).

Especie

Regiones biogeográficas

Andes, Pacífica, valle del río Cauca

Heteropterys occidentalis Cuatrec.

Heteropterys olivacea (Cuatrec.) W.R. Anderson*

Heteropterys orinocensis (Kunth) A. Juss.

Heteropterys prunifolia (Kunth) W.R. Anderson

Heteropterys quetepensis Steyerm.

Heteropterys racemosa A. Juss.

Heteropterys riparia Cuatrec.

Heteropterys rudasii W.R. Anderson*

Heteropterys siderosa Cuatrec.

Heteropterys uribei Cuatrec.

Hiraea affinis Miq.

Hiraea apaporiensis Cuatrec.

Hiraea brachyptera Triana \& Planch.

Hiraea cephalotes Triana \& Planch.

Hiraea colombiana C.V. Morton

Hiraea crassipes A. Juss.

Hiraea escobariae C.E. Anderson*

Hiraea fagifolia (DC.) A. Juss.

Hiraea faginea (Sw.) Nied.

Hiraea ferruginea Cuatrec.*

Hiraea fimbriata W.R. Anderson

Hiraea guapecita Cuatrec.

Hiraea holmgreniorum C.E. Anderson

Hiraea hypoleuca W.R. Anderson

Hiraea idroboana Cuatrec.*

Hiraea klugii Cuatrec.

Hiraea mutisiana Cuatrec.*

Hiraea opulifolia (Rusby) Nied.*

Hiraea pachypoda Nied.

Hiraea putumayensis C.V. Morton \& Cuatrec.

Hiraea reclinata Jacq.

Hiraea sanctae-marthae C.V. Morton*

Hiraea schultesii Cuatrec.

Hiraea sclerophylla Cuatrec.*

Hiraea smilacina Standl.

Hiraea ternifolia (Kunth) A. Juss.

Hiraea transiens Nied.*

Hiraea villosa Nied.

Jubelina grisebachiana W.R. Anderson

Jubelina magnifica W.R. Anderson

Jubelina uleana (Nied.) Cuatrec.

Jubelina wilburii W.R. Anderson

Lophanthera longifolia (Kunth) Griseb.

Lophanthera pendula Ducke

Lophopterys inpana W.R. Anderson
Amazonia, Guayana

Amazonia, Guayana, Orinoquia

Caribe, Sierra Nevada de Santa Marta, valle del río

Magdalena

Orinoquia

Amazonia, Guayana

Amazonia, Orinoquia

Amazonia

Amazonia, Guayana

Amazonia, Andes

Amazonia, Guayana

Amazonia, Guayana

Pacífica

Andes

Andes

Amazonia

Andes

Amazonia, Orinoquia, Pacífica, valle del río

Magdalena

Guayana, Pacífica

Andes

Orinoquia

Pacífica

Amazonia

Andes

Andes, Guayana

Amazonia

Andes

Sierra Nevada de Santa Marta

Andes

Amazonia

Caribe, Pacífica, Sierra Nevada de Santa Marta, valle

del río Magdalena

Caribe

Amazonia

Valle del río Magdalena

Amazonia, Andes

Andes, Caribe, Guayana, Orinoquia, valle del río

Magdalena

Andes, Pacífica

Valle del río Magdalena

Amazonia

Guayana

Amazonia

Valle del río Cauca, valle del río Magdalena

Amazonia, Orinoquia

Guayana

Guayana
Gradiente altitudinal

(m s.m.)

500-1500

$0-500$

$0-500$

0-1000

0-500

$0-500$

0-500

0-500

0-500

$0-2000$

$0-500$

$0-500$

0-500

1000-2000

2000-3000

$0-500$

1000-2000

0-1000

0-500

1500-2000

$0-500$

$0-500$

$0-500$

500-2000

1000-2000

$0-500$

1500-2500

1000-1500

500-2000

0-500

0-1000

0-500

$0-500$

$0-500$

$0-2500$

0-3000

0-1500

0-500

$0-500$

$0-500$

0-500

0-1000

0-500

$0-500$

$0-500$ 
Tabla 1. (Continuación.).

\begin{tabular}{|c|c|c|}
\hline Especie & Regiones biogeográficas & $\begin{array}{l}\text { Gradiente } \\
\text { altitudinal } \\
\text { (m s.m.) }\end{array}$ \\
\hline Malpighia albiflora (Cuatrec.) Cuatrec. & Pacífica & $0-500$ \\
\hline Malpighia emarginata DC. & $\begin{array}{l}\text { Andes, Caribe, Orinoquia, valle del río Cauca, valle } \\
\text { del río Magdalena }\end{array}$ & $0-2000$ \\
\hline Malpighia glabra L. & $\begin{array}{l}\text { Andes, Caribe, Pacífica, valle del río Cauca, valle } \\
\text { del río Magdalena }\end{array}$ & $0-2500$ \\
\hline Malpighia romeroana Cuatrec. & Pacífica & $0-500$ \\
\hline Malpighiodes liesneri (W.R. Anderson) W.R. Anderson & Guayana & $0-500$ \\
\hline Mascagnia allopterys (Moris) W.R. Anderson & Caribe, valle del río Magdalena & $0-500$ \\
\hline Mascagnia arenicola C.E. Anderson & $\begin{array}{l}\text { Amazonia, Andes, Orinoquia, Pacífica, valle del río } \\
\text { Magdalena }\end{array}$ & $0-1500$ \\
\hline Mascagnia conformis W.R. Anderson & Amazonia, Orinoquia & $0-500$ \\
\hline Mascagnia cynanchifolia Griseb. & Guayana & $0-500$ \\
\hline Mascagnia dissimilis C.V. Morton \& Moldenke & $\begin{array}{l}\text { Andes, Guayana, valle del río Cauca, valle del río } \\
\text { Magdalena }\end{array}$ & $0-1500$ \\
\hline Mascagnia divaricata (Kunth) Nied. & $\begin{array}{l}\text { Amazonia, Caribe, Orinoquia, Pacífica, valle del río } \\
\text { Cauca }\end{array}$ & $0-500$ \\
\hline Mascagnia eggersiana (Nied.) W.R. Anderson & Amazonia, Orinoquia & $0-500$ \\
\hline Mascagnia loretensis Morton & Amazonia & $0-500$ \\
\hline Mascagnia lugoi W.R. Anderson & Amazonia & $0-500$ \\
\hline Mascagnia macradena (DC.) Nied. & Caribe, Orinoquia, valle del río Cauca & $0-1000$ \\
\hline Mascagnia ovatifolia (Kunth) Griseb. & Caribe, Orinoquia, Pacífica, valle del río Magdalena & $0-1000$ \\
\hline Mascagnia pittieri C.V. Morton* & Andes & $1000-2000$ \\
\hline Mascagnia tenuifolia Nied. & Amazonia & $0-500$ \\
\hline Mascagnia violacea (Triana \& Planch.) Nied.* & Andes, valle del río Magdalena & $0-1500$ \\
\hline Mascagnia sp. nov.* & Pacífica & $0-500$ \\
\hline Mezia includens (Benth.) Cuatrec. & Amazonia, Guayana & $0-500$ \\
\hline Mezia rufa W.R. Anderson & Guayana & $0-500$ \\
\hline Niedenzuella castanea (Cuatrec.) W.R. Anderson & Amazonia & $0-500$ \\
\hline Niedenzuella metensis (Cuatrec.) W.R. Anderson* & Orinoquia & $0-500$ \\
\hline Niedenzuella poeppigiana (A. Juss.) W.R. Anderson & Amazonia & $0-500$ \\
\hline Niedenzuella stannea (Griseb.) W.R. Anderson & Amazonia, valle del río Magdalena & $0-500$ \\
\hline Pterandra colombiana C.E. Anderson* & Valle del río Cauca, valle del río Magdalena & $0-1000$ \\
\hline Pterandra ultramontana Cuatrec.* & Pacífica & $0-500$ \\
\hline Spachea herbert-smithii (Rusby) Cuatrec.* & $\begin{array}{l}\text { Andes, Caribe, Sierra Nevada de Santa Marta, valle } \\
\text { del río Cauca, valle del río Magdalena }\end{array}$ & $0-1500$ \\
\hline Spachea membranacea Cuatrec. & Pacífica & $0-500$ \\
\hline Spachea tricarpa A. Juss. & Amazonia & $0-500$ \\
\hline Stigmaphyllon adenodon A. Juss. & Amazonia & $0-500$ \\
\hline Stigmaphyllon alternans Triana \& Planch. & Amazonia, Orinoquia & $0-500$ \\
\hline Stigmaphyllon bannisterioides (L.) C.E. Anderson & Caribe, Pacífica & $0-500$ \\
\hline Stigmaphyllon bogotense Triana \& Planch. & $\begin{array}{l}\text { Andes, Sierra Nevada de Santa Marta, valle del río } \\
\text { Cauca, valle del río Magdalena }\end{array}$ & $0-3500$ \\
\hline Stigmaphyllon cardiophyllum A. Juss. & Amazonia & $0-500$ \\
\hline Stigmaphyllon ciliatum (Lam.) A. Juss. & Pacífica & $0-500$ \\
\hline Stigmaphyllon columbicum Nied. & $\begin{array}{l}\text { Andes, Caribe, Pacífica, Sierra Nevada de Santa } \\
\text { Marta, valle del río Cauca, valle del río Magdalena }\end{array}$ & $0-3500$ \\
\hline Stigmaphyllon dichotomum (L.) Griseb. & $\begin{array}{l}\text { Andes, Caribe, Orinoquia, Pacífica, valle del río } \\
\text { Cauca, valle del río Magdalena }\end{array}$ & $0-1500$ \\
\hline Stigmaphyllon echitoides Triana \& Planch. & $\begin{array}{l}\text { Andes, Pacífica, valle del río Cauca, valle del río } \\
\text { Magdalena }\end{array}$ & $0-2500$ \\
\hline
\end{tabular}


Tabla 1. (Continuación.).

Especie

Stigmaphyllon ellipticum (Kunth) A. Juss.

Stigmaphyllon florosum C.E. Anderson

Stigmaphyllon goudotii C.E. Anderson*

Stigmaphyllon herbaceum Cuatrec.

Stigmaphyllon hypargyreum Triana \& Planch.

Stigmaphyllon lacunosum A. Juss.

Stigmaphyllon lindenianum A. Juss.

Stigmaphyllon maynense Huber

Stigmaphyllon orientale Cuatrec.*

Stigmaphyllon puberum (Rich.) A. Juss.

Stigmaphyllon romeroi Cuatrec.*

Stigmaphyllon sarmentosum Cuatrec.

Stigmaphyllon singulare C.E. Anderson

Stigmaphyllon sinuatum (DC.) A. Juss.

Stigmaphyllon stenophyllum C.E. Anderson*

Stigmaphyllon suffruticosum Cuatrec.*

Stigmaphyllon tergolanatum Cuatrec.*

Stigmaphyllon velutinum Triana \& Planch.*

Stigmaphyllon venulosum Cuatrec.*

Tetrapterys acapulcensis Kunth

Tetrapterys amazonica C.E. Anderson

Tetrapterys benthamii Triana \& Planch.*

Tetrapterys callejasii W.R. Anderson3*

Tetrapterys calophylla A. Juss.

Tetrapterys chloroptera Cuatrec.

Tetrapterys crispa A. Juss.

Tetrapterys diptera Cuatrec.

Tetrapterys discolor (G. Mey.) DC.

Tetrapterys goudotiana Triana \& Planch.

Tetrapterys hirsutula Cuatrec. \& Croat

Tetrapterys jamesonii Turcz.

Tetrapterys magnifolia Griseb.

Tetrapterys mucronata Cav.

Tetrapterys natans W.R. Anderson

Tetrapterys nitida A. Juss.

Tetrapterys papyracea Triana \& Planch.*

Tetrapterys seemannii Triana \& Planch.

Tetrapterys splendens Cuatrec.*

Tetrapterys steyermarkii W.R. Anderson

Tetrapterys subaptera Cuatrec.

Tetrapterys tinifolia Triana \& Planch.

Tetrapterys tolimensis Sprague*

Tetrapterys sp. nov. 1*

Tetrapterys sp. nov. 2
Regiones biogeográficas

Gradiente

altitudinal

(m s.m.)

$0-1500$

Andes, Caribe, Pacífica

$0-500$

Amazonia

Andes

$1000-1500$

Caribe, Pacífica, valle del río Cauca, valle del río

Magdalena

$0-1000$

Andes, Pacífica

$0-2500$

$0-500$

\begin{tabular}{l|l}
\hline Amazonia & $0-500$ \\
\hline Pacífica & $0-500$
\end{tabular}

\begin{tabular}{lr}
\hline Amazonia & $0-500$ \\
\hline
\end{tabular}

\begin{tabular}{l|l}
\hline Orinoquia & $0-500$
\end{tabular}

Amazonia, Guayana, Orinoquia, Pacífica $\quad 0-500$

Caribe

Andes

Andes

$0-500$

2000-2500

1000-1500

Amazonia, Guayana, Orinoquia, valle del río Cauca,

valle del río Magdalena

$0-1000$

Andes

2000-2500

Andes, valle del río Cauca

$500-1500$

Valle del río Magdalena

$500-1000$

Andes, valle del río Magdalena

$500-2500$

Andes, Orinoquia

$0-3000$

Andes, valle del río Cauca, valle del río Magdalena

$0-2000$

Amazonia

$0-500$

Andes

$500-2500$

Andes, Caribe, Pacífica, valle del río Cauca, valle

del río Magdalena

$0-2000$

Amazonia, Andes, Orinoquia

$0-1500$

Amazonia, Guayana, Orinoquia, valle del río Cauca

$0-1000$

Andes, Caribe, Orinoquia, valle del río Magdalena

$0-1500$

Andes, Pacífica, valle del río Magdalena

$0-2000$

Amazonia, Andes, Orinoquia, valle del río Cauca,

valle del río Magdalena

$0-2000$

Amazonia, Caribe, Pacífica, valle del río Magdalena

$0-1000$

Pacífica

$0-500$

Andes

$1500-3000$

Valle del río Cauca, valle del río Magdalena

$0-500$

Amazonia, Guayana, Orinoquia, Pacífica

$0-500$

Amazonia, Guayana

$0-500$

Amazonia

$0-500$

Amazonia, Andes, Guayana, Orinoquia

$0-1000$

Caribe, Pacífica

$0-500$

Andes, valle del río Magdalena

$500-2500$

Caribe

$0-500$

Pacífica

$0-500$

Amazonia, Andes, Pacífica, valle del río Magdalena

$0-2500$

Valle del río Magdalena

$0-500$

Amazonia

$0-500$

Amazonia

$0-500$


Tabla 2. Riqueza y endemismo de las Malpighiaceae en Colombia, sobre la base de gradientes altitudinales. *: La suma de las columnas no es aritmética, ya que hay varias especies que se distribuyen en más de un intervalo altitudinal.

\begin{tabular}{|c|c|c|c|c|c|}
\hline $\begin{array}{c}\text { Intervalo altitudinal } \\
\text { (m s.m.) }\end{array}$ & $\begin{array}{c}\text { Número de } \\
\text { especies }\end{array}$ & $\begin{array}{c}\text { Número de especies } \\
\text { endémicas }\end{array}$ & $\begin{array}{c}\text { Número de especies } \\
\text { arbóreas o arbustivas }\end{array}$ & $\begin{array}{c}\text { Número de especies } \\
\text { lianescentes }\end{array}$ & $\begin{array}{c}\text { Número de especies } \\
\text { sufrútices }\end{array}$ \\
\hline $0-500$ & 212 & 28 & 71 & 140 & 1 \\
\hline $500-1000$ & 94 & 17 & 25 & 67 & 1 \\
\hline $1000-1500$ & 72 & 17 & 22 & 49 & 1 \\
\hline $1500-2000$ & 53 & 13 & 16 & 36 & 1 \\
\hline $2000-2500$ & 30 & 9 & 10 & 20 & 0 \\
\hline $2500-3000$ & 10 & 1 & 3 & 7 & 0 \\
\hline $3000-3200$ & 2 & 0 & 0 & 2 & \\
\hline Total* & 246 & 47 & 83 & 162 & \\
\hline
\end{tabular}

Asimismo, el número de especies endémicas por intervalo de altitud muestra el mismo patrón, en donde las tierras bajas (0-500 m s.m.) son las que presentan el mayor número de especies endémicas (28), seguido del segundo intervalo altitudinal con 17 especies y el endemismo va disminuyendo a medida que se asciende en el gradiente altitudinal (Tabla 2). Estos datos se ajustan a los encontrados para otras familias vegetales en Colombia (Rangel-Ch., 2006; 2015; Giraldo-Cañas, 2010; 2018) estudiadas en un contexto de gradientes altitudinales, así como en Ecuador (Lægaard, 1999; Kessler, 2002) y Venezuela (Giraldo-Cañas, 2014), en las cuales las tierras bajas y medias (0$1500 \mathrm{~m}$ s.m.) tienen la mayor riqueza, no sólo en número de especies sino también en géneros y clados (Giraldo-Cañas, 2014).

El patrón de riqueza altitudinal, así como el patrón de endemismo altitudinal, muestran una disminución con la altitud $(y=-0,061 x+173,57$; $\mathrm{R}^{2}=0,82 ; y=-0,009 x+27,76 ; \mathrm{R}^{2}=0,95$, respectivamente, donde " $y$ " es el número de especies y " $x$ " la altitud). Este patrón de riqueza de especies es exhibido por otros grupos vegetales en el neotrópico, tales como Poaceae (Giraldo-Cañas, 2010; 2014), Marcgraviaceae (Giraldo-Cañas, 2018), así como por la riqueza de especies en numerosos bosques neotropicales en diferentes gradientes altitudinales (Gentry, 1995). Por otra parte, los datos aquí analizados para las Malpighiaceae de Colombia, no se ajustan al "efecto del dominio medio" (Colwell \& Lees, 2000; Adams, 2009; Giraldo-Cañas, 2014), el cual contempla que la mayor riqueza de especies, en un contexto de gradientes altitudinales, se encuentra en las alturas intermedias, como es el caso de la riqueza de Acanthaceae, Bromeliaceae, Pteridophyta, Solanaceae) (Kessler \& Kluge, 2008; Adams, 2009) o el caso de las especies de la familia Frullaniaceae de las hepáticas en áreas montanas neotropicales (Gradstein \& Uribe, 2011). Esta situación se ajusta a la naturaleza ecológica y biogeográfica de la mayoría de las especies de Malpighiaceae, las cuales prefieren los hábitats húmedos $\mathrm{y}$ cálidos de tierras bajas, principalmente de la cuenca amazónica, de variadas formaciones vegetales de los escudos precámbricos sudamericanos y de la Mata Atlántica (Anderson, 1981; 2001; Mamede et al., 2010; Rangel-Ch., 2015; Almeida et al., 2016).

Los límites altitudinales de la familia Malpighiaceae en Colombia, alcanzan los 3200 m s.m. y tan sólo diez especies se distribuyen en las tierras altas (2500-3200 m s.m.), como en algunos bosques andinos, tanto primarios como secundarios, arbustales, bordes de bosque y en zonas de transición entre bosques altoandinos y páramos, a saber, Bronwenia cornifolia ( $\leq 3000$ m s.m.), Bunchosia armeniaca $(\leq 2800 \mathrm{~m}$ s.m.), Bunchosia cestrifolia $(\leq 2950 \mathrm{~m} \quad$ s.m.), Bunchosia sp. nov. ( $\leq 2700 \mathrm{~m}$ s.m.), Hiraea colombiana ( $\leq 2800$ m s.m.), Hiraea ternifolia ( $\leq 2750$ m s.m.), Stigmaphyllon bogotense $(\leq 3200 \mathrm{~m} \quad$ s.m.), Stigmaphyllon columbicum $(\leq 3200 \mathrm{~m}$ s.m.), Stigmaphyllon venulosum ( $\leq 2800 \mathrm{~m}$ s.m.) y Tetrapterys jamesonii ( $\leq 2600 \mathrm{~m}$ s.m.) (Tabla 1).

La Amazonia, los Andes y el valle del río Magdalena, exhiben la riqueza de especies más alta, con 116, 89 y 68, respectivamente (Figura 1, Tabla 3). 


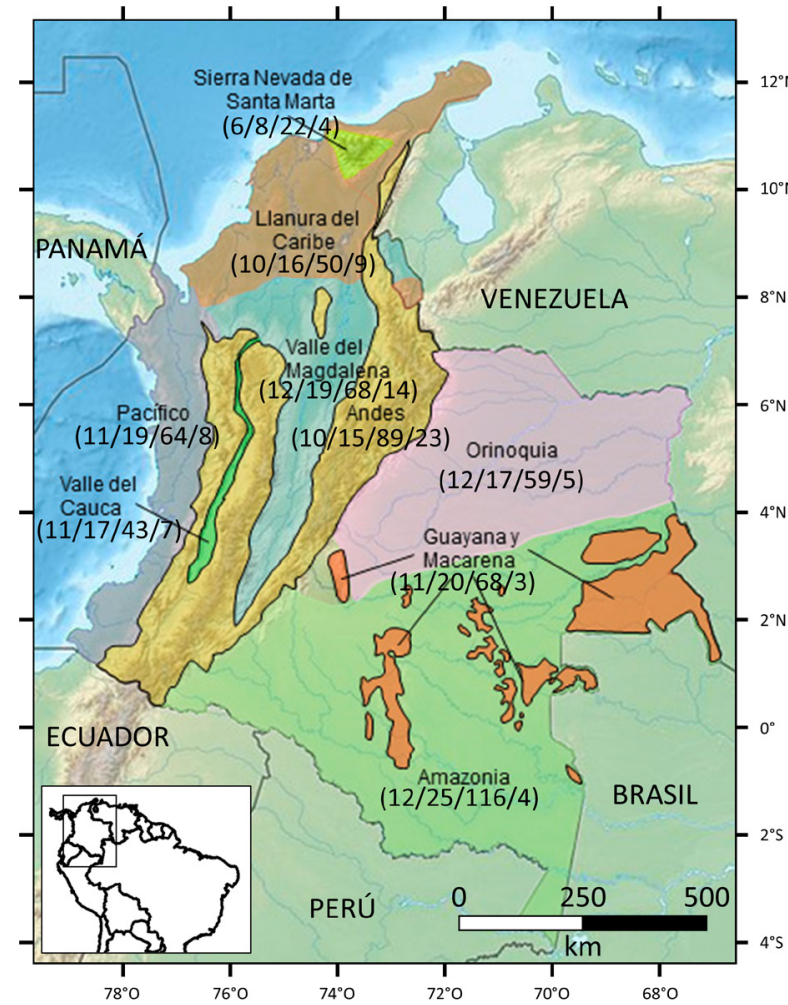

Fig. 1. Diversidad filogenética, riqueza genérica $y$ específica y endemismo de las Malpighiaceae en las regiones biogeográficas de Colombia (número de clados/ número de géneros/número de especies/número de especies endémicas). En esta figura, por cuestiones de escala, no aparecen las islas caribeñas colombianas (2/2/4/0) (mapa modificado de Bernal et al., 2016). Figura en color en la versión en línea http:/www.ojs.darwin.edu.ar/index.php/ darwiniana/article/view/923/1206

Cabe resaltar que en la Sierra Nevada de Santa Marta (la montaña costera más alta del mundo, Alvear et al., 2015), sólo se han registrado 22 especies, pero claramente esta baja riqueza se debe a que dicha sierra está pobremente muestreada (Alvear et al., 2015), así que se cree que su real riqueza biológica y endemismo, no sólo de Malpighiaceae sino de muchos otros grupos de plantas, sería muy destacada (Alvear et al., 2015), y esto se basa en la "Hipótesis de la heterogeneidad ambiental", la cual sustenta la idea de que ambientes heterogéneos permiten ser colonizados por un mayor número de especies, con lo cual aumentan la riqueza y la diversidad biológicas (Badii et al., 2007).
El mayor número de especies endémicas se encuentra en los Andes y en el valle del río Magdalena, con 23 y 14 especies, respectivamente (Figura 1, Tabla 3). Es curioso el bajo endemismo en la Guayana colombiana, toda vez que las Malpighiaceae presentan una riqueza y un endemismo muy destacados en toda esta región biogeográfica (Anderson, 1981), con 160 especies, 100 de las cuales son endémicas (Anderson, 1981; Giraldo-Cañas, 2001; 2008), y más concretamente, la Guayana venezolana presenta 153 especies, 32 de las cuales son endémicas (Anderson, 2001). Así, al igual que para la Sierra Nevada de Santa Marta, los inventarios vegetales son escasos y fragmentarios en la Guayana colombiana (así como en otras áreas colombianas, e. g. Amazonia, Pacífica y varios valles intra-andinos, entre otras), por lo tanto, estos datos actuales de riqueza y endemismo, no mostraría su real expresión en esta área. Adicionalmente, las formaciones vegetales de la Guayana se asientan en sustratos muy antiguos, ya que dicha región es de origen precámbrico (véanse Giraldo-Cañas, 2001; 2008) y así, en este contexto se podría recurrir a la "Hipótesis del Tiempo" para esperar una mayor riqueza y diversidad de especies en la Guayana colombiana, dado que esta hipótesis relaciona antigüedad geológica con una mayor riqueza y diversidad biológicas (Badii et al., 2007).

Disyunciones anfiatlánticas. De las especies presentes en Colombia, Heteropterys leona y Stigmaphyllon bannisterioides exhiben una distribución natural en América tropical y en la costa atlántica de África tropical (Anderson \& Anderson, 2016). Estas especies poseen frutos adaptados a la dispersión hidrócora, como la presencia de aerénquima en las paredes del fruto, lo cual le permite una mayor flotabilidad (Anderson, 2001). Así, recientemente estas dos especies pudieron haber llegado a África por medio del océano Atlántico (Davis \& Anderson, 2010).

Disyunciones neotropicales. Entre las especies presentes en Colombia, hay algunas que muestran disyunciones intra-americanas, como por ejemplo Byrsonima bucidifolia (presente en la isla caribeña colombianadeProvidencia, asícomoenlapenínsulade Yucatán, México), Ectopopterys soejartoi (conocida en Colombia únicamente en el norte de Antioquia, así como en algunas áreas de Ecuador y Perú), 
Tabla 3. Riqueza de las Malpighiaceae en las regiones biogeográficas de Colombia. *: La suma de las columnas no es aritmética, ya que hay varios clados, géneros y especies que se distribuyen en más de una región biogeográfica.

\begin{tabular}{|l|c|c|c|c|}
\hline \multicolumn{1}{|c|}{ Región biogeográfica } & Número de clados & Número de géneros & Número de especies & Número de especies endémicas \\
\hline Amazonia & 12 & 25 & 116 & 4 \\
\hline Andes & 10 & 15 & 89 & 23 \\
\hline Guayana & 11 & 20 & 68 & 3 \\
\hline Islas Caribeñas & 2 & 2 & 4 & 9 \\
\hline Caribe & 10 & 16 & 50 & 5 \\
\hline Orinoquia & 12 & 17 & 59 & 8 \\
\hline Pacífica & 11 & 19 & 64 & 4 \\
\hline Sierra Nevada de Santa Marta & 6 & 8 & 22 & 7 \\
\hline Valle del río Cauca & 11 & 17 & 43 & 14 \\
\hline Valle del río Magdalena & 12 & 19 & 68 & 47 \\
\hline \multicolumn{1}{|r|}{ Total* } & 15 & 34 & 246 & \\
\hline
\end{tabular}

Hiraea villosa (conocida de algunas áreas colombianas del valle del río Magdalena, así como en algunas áreas del Perú), Stigmaphyllon bogotense (conocida únicamente de los Andes septentrionales y de Costa Rica), Stigmaphyllon sarmentosum (conocida únicamente del departamento colombiano de Cundinamarca en el centro del país, así como en algunas áreas de Ecuador y nordeste del Perú) y Tetrapterys magnifolia (conocida de algunas áreas de los departamentos de Antioquia y Cesar, en el norte de Colombia, así como en algunas áreas de Ecuador y Perú) (Anderson \& Anderson, 2016). Estas disyunciones se deben analizar con cautela, ya que son evidentes los vacíos de inventarios florísticos en muchas áreas de Colombia. Así, esta distribución aparentemente disyunta quizás se deba a problemas y vacíos de muestreos e inventarios en varias regiones colombianas, como se mostrará más adelante.

\section{Diversidad filogenética de las Malpighiaceae} en Colombia. En Colombia están representados 15 de los 19 clados reconocidos para la familia (véase Anderson et al., 2020), con 34 de los 77 géneros descritos (tablas 3 y 4). Los clados más diversificados en Colombia son el clado Stigmaphyllon con cinco géneros, 48 especies, diez de las cuales son endémicas de Colombia (5/48/10), el clado Byrsonima (3/39/5), el clado Hiraea (3/31/9), el clado Heteropterys $(1 / 28 / 3)$, el clado Tetrapterys s. str. $(2 / 27 / 6)$ y el clado Bunchosia (1/23/5) (Tabla 4). La diversidad filogenética en los gradientes altitudinales es similar al patrón de la riqueza específica por intervalo altitudinal, ya que las regiones naturales referidas a las tierras bajas (Amazonia, Orinoquia, valle del río Magdalena, Guayana, Pacífica, valle del río Cauca, Caribe), son las que presentan la mayor diversidad en clados. Si bien la Amazonia es la región más rica en especies, presenta el mismo número de clados (12) que la Orinoquia (una región que tiene la mitad de la riqueza específica de la Amazonia) y que el valle del río Magdalena; le siguen en cantidad de clados, la Guayana, la Pacífica y el valle del río Cauca, con once clados cada una (Tabla 3). A pesar de ser los Andes la segunda región natural más rica en especies (89), es la que ocupa el séptimo lugar en cuanto al número de clados (diez de los quince clados registrados en Colombia, faltando tanto clados basales como más recientes) (Tabla 3). Estas cifras son diferentes a las presentadas por Anderson \& Anderson (2016), toda vez que aquí se amplía el número de géneros para Colombia, de 32 a 34 , y el número de especies y por ende, de clados para varias unidades biogeográficas colombianas, como por ejemplo, pasar de diez especies para la Sierra Nevada de Santa Marta a 22, pasar de 57 especies para la Guayana a 68, de 42 especies para el valle del río Cauca a 43 y de 67 especies para el valle del río Magdalena a 68 especies, además, se actualizaron algunos datos para la distribución altitudinal de varias especies. No obstante, con nuevas exploraciones en varias áreas del territorio nacional, se esperaría que la Guayana colombiana presente el mayor número de especies y de clados, seguida, probablemente, por la Amazonia, ya que en ambas regiones sudamericanas se encuentran la mayor riqueza de especies y la mayor cantidad de linajes. 
Tabla 4. Diversidad filogenética de las Malpighiaceae y su representatividad en Colombia. La configuración de los clados y su distribución geográfica están basadas en Anderson et al. (2020). *: Género presente en Colombia. Los números entre paréntesis corresponden al número de especies por género presentes en Colombia (totales/endémicas).

\begin{tabular}{|c|c|c|}
\hline Clado & Géneros & Distribución \\
\hline Acmanthera & Acmanthera, Coleostachys, Pterandra* $(2 / 2)$ & Sudamérica y Panamá \\
\hline Acridocarpus & Acridocarpus, Brachylophon & Paleotrópico \\
\hline Amorimia & Amorimia* $(1 / 1)$ & Sudamérica \\
\hline Barnebya & Barnebya & Brasil \\
\hline Bunchosia & $\begin{array}{l}\text { Bunchosia* }(23 / 5), \text { Echinopterys, Heladena, } \\
\text { Henleophytum, Thryallis, Tristellateia }\end{array}$ & Neotrópico y paleotrópico \\
\hline Byrsonima & Blepharandra* $(2 / 0)$, Byrsonima* $(36 / 5)$, Diacidia* $(1 / 0)$ & $\begin{array}{l}\text { El Caribe y desde el sudeste de la Florida hasta el } \\
\text { sudeste de Brasil }\end{array}$ \\
\hline Carolus & $\begin{array}{l}\text { Carolus* }(1 / 0), \text { Dicella* } \\
(1 / 0), \text { Flabellariopsis, Hiptage, Tricomaria }\end{array}$ & Neotrópico, paleotrópico y sudeste asiático \\
\hline Christianella & $\begin{array}{l}\text { Alicia* }(1 / 0), \text { Callaeum } *(1 / 0), \text { Christianella* } \\
(2 / 0), \text { Flabellaria, Jubelina* }(4 / 0), \text { Malpighiodes* } \\
(1 / 0), \text { Mezia }^{*}(2 / 0)\end{array}$ & Desde Texas hasta el sur de Sudamérica y África \\
\hline Ectopopterys & Ectopopterys* $(1 / 0)$ & Colombia (Antioquia), Ecuador y Perú \\
\hline Galphimia & $\begin{array}{l}\text { Andersoniodoxa* }(2 / 1), \text { Galphimia, Lophanthera* } \\
(2 / 0), \text { Spachea* }(3 / 1), \text { Verrucularia }\end{array}$ & Desde Texas hasta Sudamérica, así como en Cuba \\
\hline Heteropterys & Heteropterys* $(28 / 3)$ & $\begin{array}{l}\text { Desde el norte de México hasta el sudeste de Brasil } \\
\text { y norte de la Argentina, el Caribe y la costa atlántica } \\
\text { de África }\end{array}$ \\
\hline Hiraea & $\begin{array}{l}\text { Adelphia* }(2 / 1), \text { Excentradenia, Hiraea* } \\
(28 / 8), \text { Lophopterys } *(1 / 0), \text { Psychopterys }\end{array}$ & Neotrópico \\
\hline Malpighia & $\begin{array}{l}\text { Aspidopterys, Calcicola, Caucanthus, Diaspis, } \\
\text { Digoniopterys, Madagaskaria, Malpighia* }(4 / 0), \\
\text { Mascagnia* (15/3), Microsteira, Rhynchophora, Triaspis }\end{array}$ & Neotrópico y paleotrópico \\
\hline Mcvaughia & Burdachia* (2/0), Glandonia* (1/0), Mcvaughia & Sudamérica \\
\hline Niedenzuella & Niedenzuella* $(4 / 1)$ & Neotrópico \\
\hline Ptilochaeta & Dinemagonum, Dinemandra, Lasiocarpus, Ptilochaeta & México, Argentina, Brasil, Chile y Paraguay \\
\hline Stigmaphyllon & $\begin{array}{l}\text { Aspicarpa, Banisteriopsis* }(6 / 0), \text { Bronwenia* }(3 / 0), \\
\text { Camarea, Cordobia, Cottsia, Diplopterys* }(10 / 1), \text { Gallardoa, } \\
\text { Gaudichaudia* }(1 / 0), \text { Janusia, Mionandra, Peixotoa, } \\
\text { Philgamia, Sphedamnocarpus, Stigmaphyllon* }(28 / 9)\end{array}$ & $\begin{array}{l}\text { Nuevo Mundo (desde Texas hasta la Argentina, Bolivia } \\
\text { y Paraguay) y Viejo Mundo (África subsahariana, } \\
\text { Madagascar, Australia, Filipinas, Taiwán, Indonesia, } \\
\text { Nueva Caledonia y otras islas del Pacífico) }\end{array}$ \\
\hline Tetrapterys s. s. & Glicophyllum* (2/0), Tetrapterys* (25/6) & Neotrópico \\
\hline Tetrapterys s. 1. & Tetrapterys $(0 / 0)$ & Neotrópico \\
\hline
\end{tabular}

\section{CONCLUSIONES}

Colombia es el segundo país más rico en Malpighiaceae del mundo (34 géneros, 246 especies y un endemismo del 19,1\%), después de Brasil [44 géneros, 584 especies y un $61 \%$ de endemismo (Mamede et al. 2010; Almeida et al., 2021)]. No obstante, son evidentes los pocos muestreos botánicos en muchas áreas colombianas (GiraldoCañas, obs. pers. realizadas durante casi 30 años de exploración en Colombia), por lo tanto, persisten los vacíos en torno de la riqueza biológica del país, ya que falta por explorar cerca de un $40 \%$ del territorio colombiano (Giraldo-Cañas, obs. pers.). En este sentido, son prioritarias las expediciones a la Sierra Nevada de Santa Marta (en el Caribe), la Guayana (principalmente en los departamentos de Caquetá, Guainía y Vaupés), la Orinoquia (principalmente en los departamentos de Arauca y Vichada), la región Pacífica (incluidas varias de sus serranías), la región del Catatumbo (Norte de Santander), varias zonas de las vertientes andinas (principalmente los cañones intra-andinos) y a pesar de que la Amazonia colombiana es la más rica, es evidente la necesidad de explorar varias áreas amazónicas de los departamentos del Amazonas, Caquetá, Guaviare y Putumayo. Es necesario destacar que gran parte de estas áreas estaban "prohibidas" para 
la investigación científica, debido a la guerra interna colombiana, la cual duró cerca de 55 años, y que tras la reciente firma de un acuerdo de paz están "abiertas" a variadas iniciativas de exploración e investigación (Baptiste et al., 2017).

El patrón de riqueza de especies, endemismo y diversidad filogenética de las Malpighiaceae en el contexto altitudinal colombiano muestra una alta riqueza en las tierras bajas. Este patrón coincide con varias familias, especialmente diversificadas en las tierras bajas neotropicales (0-1500 m s.m.), como Annonaceae, Apocynaceae, Arecaceae, Bignoniaceae, Chrysobalanaceae, Convolvulaceae, Euphorbiaceae, Fabaceae, Lamiaceae, Malvaceae, Marantaceae, Marcgraviaceae, Moraceae, Myristicaceae, Myrtaceae, Passifloraceae, Piperaceae, Poaceae, Rubiaceae, Sapotaceae, entre otras (RangelCh., 2006; 2015; Bernal et al., 2016). No obstante, este comportamiento es diferente al exhibido por otros grupos vegetales, los cuales muestran un patrón correspondiente al "efecto del dominio medio" (Acanthaceae, Araceae, Bromeliaceae, Ericaceae, Gesneriaceae, hepáticas, líquenes, Orchidaceae, Pteridophyta, Solanaceae), toda vez que su mayor riqueza de especies se alcanza en altitudes intermedias (1500-2500 m s.m.) (Rangel-Ch., 2006; 2015; Bernal et al., 2016) y contrario a otras familias, las cuales alcanzan su riqueza más destacada en alturas mayores ( $\geq 2500 \mathrm{~m} \mathrm{s.m}$.), como en el caso de varios grupos (tribus, géneros) de las familias Apiaceae, Asteraceae, Calceolariaceae, Cunoniaceae, Hypericaceae, Juncaceae, Orchidaceae, Plantaginaceae, Poaceae, Ranunculaceae, Rosaceae, entre otras (Rangel-Ch., 2006; 2015; Bernal et al., 2016).

La consideración de regiones con elevada riqueza de especies, endemismo destacado y una apreciable diversidad filogenética, junto con la combinación de la representatividad de otros grupos biológicos, tanto vegetales como animales, podrían constituir insumos para definir y delimitar nuevas áreas prioritarias para la conservación en Colombia, y varias de estas regiones no cuentan con la suficiente representatividad de áreas biogeográficas con categorías de conservación en el "Sistema Nacional Ambiental". Por otra parte, se recomienda evaluar, en un futuro cercano, el recambio, la abundancia, el desplazamiento y la extinción de especies en intervalos altitudinales, con miras a proporcionar nuevas evidencias en torno de los efectos del calentamiento climático global.

\section{AGRADECIMIENTOS}

A la Universidad Nacional de Colombia por todas las facilidades brindadas para la realización de este trabajo. A los curadores de los herbarios ACAM, AFP, ANDES, AS, BA, BAF, BRG, CAUP, CDMB, CEN, CEPEC, COAH, COL, CR, CUVC, CHOCO, EAN, F, FAUC, FMB, G, HECASA, HFAB, HORI, HPUJ, HUA, HUC, HUQ, IAN, IBGE, INPA, IPA, JAUM, LLANOS, MEDEL, MO, NY, PSO, QCA, RSA, SI, SURCO, TOLI, UDBC, UIS, UPTC, US y VALLE, por todo su respaldo y por permitir el estudio de sus colecciones. A Dairon Cárdenas (COAH) y a la Asociación Colombiana de Herbarios por financiar numerosos viajes para visitar diferentes herbarios colombianos. El mapa de las regiones biogeográficas de Colombia se modificó de Bernal et al. (2016). A Gonzalo Andrade (director del Instituto de Ciencias Naturales, Universidad Nacional de Colombia), a la Editorial UN (Universidad Nacional de Colombia) y a Rodrigo Bernal (editor del Catálogo de plantas y líquenes de Colombia), por permitir el uso del mapa de las regiones biogeográficas de Colombia. Al Comité Editorial y a los evaluadores anónimos por sus acertados y valiosos comentarios.

\section{BIBLIOGRAFÍA}

Abadía Bonilla, D.; Á. J. Lema Tapia \& L. Y. Palacios-Tello. 2015. Composición de lianas y bejucos en el Chocó biogeográfico colombiano. Rodriguésia 66: 665-673. DOI: https://doi.org/10.1590/2175-7860201566301

Acevedo-Rodríguez, P. 2005. Vines and climbing plants of Puerto Rico and the Virgin Islands. Contributions from the United States National Herbarium 51: 1-483.

Adams, J. 2009. Species richness. Patterns in the diversity of life. Berlín: Springer-Praxis Publishing.

Almeida, R. F.; A. Francener \& A. M. Amorim. 2016. A generic synopsis of Malpighiaceae in the Atlantic Forest. Nordic Journal of Botany 34: 285-301. DOI: https://doi.org/10.1111/njb.01016

Almeida, R. F.; A. Francener, C. Pessoa, R. Sebastiani, Y. R. Oliveira, A. M. A. Amorim, M. C. H. Mamede. 2021. Malpighiaceae, en Flora do Brasil 2020 em construção. Jardim Botânico do Rio de Janeiro. http://floradobrasil.jbrj.gov.br/ reflora/floradobrasil/FB155 (Consultado febrero de 2021).

Almeida, R. R. \& C. van den Berg. 2021. Molecular phylogeny and character mapping support generic adjustments in the Tetrapteroid clade (Malpighiaceae). Nordic Journal of Botany 2021: e02876. DOI: https://doi.org/10.1111/njb.02876 
Alvear, M.; G. Ocampo, C. Parra-O., E. Carbonó \& F. Almeda. 2015. Melastomataceae of the Sierra Nevada de Santa Marta (Colombia): floristic affinities and annotated catalogue. Phytotaxa 195: 1-30. DOI: https://doi.org/10.11646/phytotaxa.195.1.1

Anderson, W. R. 1981. Malpighiaceae. The botany of the Guayana Highland. Memoirs of the New York Botanical Garden 32: 21-305.

Anderson, W. R. 2001. Malpighiaceae. Flora of the Venezuelan Guayana 6: 82-185.

Anderson, W. R. 2006. Eight segregates from the Neotropical genus Mascagnia (Malpighiaceae). Novon 16: 168-204. DOI: https:// doi.org/10.3417/1055-3177(2006)16[168:ESFTNG]2.0.CO;2

Anderson, W. R. 2013. Origins of Mexican Malpighiaceae. Acta Botánica Mexicana 104: 107-156.

Anderson, W. R. 2016. Malpighiaceae. Flora of North America 12: 354-364.

Anderson, W. R. \& C. Anderson. 2016. Malpighiaceae, en R. Bernal, S. R. Gradstein \& M. Celis (eds.), Catálogo de plantas y líquenes de Colombia II: 1512-1535. Bogotá D. C.: Instituto de Ciencias Naturales, Universidad Nacional de Colombia.

Anderson, W. R. \& C. C. Davis. 2007. Generic adjustments in Neotropical Malpighiaceae. Contributions from University Michigan Herbarium 25: 137-166.

Anderson, W. R. \& C. C. Davis. 2013. Combination of Mascagnia and Triopterys (Malpighiaceae), en Memoirs of the New York Botanical Garden. Harmony and Grit: papers celebrating the Holmgrens' completion of Intermountain Flora, pp. 191-203.

Anderson, W. R.; C. Anderson \& C. C. Davis. 2020. Malpighiaceae. Herbarium, University of Michigan. http:// herbarium.lsa.umich.edu/malpigh/index.html (Consultado: noviembre de 2020).

Badii, M. H.; J. Landeros \& E. Cerna. 2007. Patrones de asociación de especies y sustentabilidad. Daena (International Journal of Good Conscience) 3: 632-660.

Baptiste, B.; M. Pinedo-Vásquez, V. H. Gutiérrez-Vélez, G. I. Andrade, P. Vieira, L. M. Estupiñán-Suárez, M. C. Londoño, W. Laurance \& T. M. Lee. 2017. Greening peace in Colombia. Nature, Ecology \& Evolution 1: 0102. DOI: https://doi.org/10.1038/s41559-017-0102

Bernal, R.; S. R. Gradstein \& M. Celis (eds.). 2016. Catálogo de plantas y líquenes de Colombia, volúmenes I y II. Bogotá D. C.: Universidad Nacional de Colombia, Facultad de Ciencias, Instituto de Ciencias Naturales.

Borges, R. A. X.; M. Machado Saavedra, J. Naoki Nakajima \& R. Campostrini Forzza. 2010. The Asteraceae flora of the Serra do Ibitipoca: analyses of its diversity and distribution compared with selected areas in Brazilian mountain ranges. Systematics and Biodiversity 8: 471-479. DOI: https://doi.or $\mathrm{g} / 10.1080 / 14772000.2010 .517573$
Colwell, K. R. \& D. C. Lees. 2000. The mid-domain efect: geometric constraints on the geography of species richness. Trends in Ecology and Evolution 15: 70-76. DOI: https:// doi.org/10.1016/s0169-5347(99)01767-x

Cuatrecasas, J. 1958. Prima Flora Colombiana. 2. Malpighiaceae. Webbia 13: 343-664.

Daniel, W. 1990. Bioestadística. 3ra. edición. México D. F.: Ed. Limusa.

Davis, C. C. \& W. R. Anderson. 2010. A complete generic phylogeny of Malpighiaceae inferred from nucleotide sequence data and morphology. American Journal of Botany 97: 2031-2048. DOI: https://doi.org/10.3732/ ajb. 1000146

Davis, C. C.; L. C. Marinho \& A. M. Amorim. 2020a. Andersoniella: a new genus of Neotropical Malpighiaceae. Harvard Papers in Botany 25: 51-56. DOI: https://doi. org/10.3100/hpib.v25iss1.2020.n6

Davis, C. C.; L. C. Marinho \& A. M. Amorim. 2020 b. Andersoniodoxa, a replacement name for Andersoniella (Malpighiaceae). Phytotaxa 470: 121-122. DOI: https://doi. org/10.11646/phytotaxa.470.1.9

Etter, A. \& W. van Wyngaarden. 2000. Patterns of landscape transformation in Colombia, with emphasis in the Andean Region. Ambio 29: 432-439. DOI: https://doi. org/10.1579/0044-7447-29.7.432

Ferro, I. \& R. M. Barquez. 2014. Patrones de distribución de micromamíferos en gradientes altitudinales del noroeste argentino. Revista Mexicana de Biodiversidad 85: 472-490. DOI: https://doi.org/10.7550/rmb.38029

Funk, V. A. 2006. Floras: A model for biodiversity studies or a thing of the past? Taxon 55: 581-588. DOI: https://doi. org/10.2307/25065635

Funk, V. A. \& K. S. Richardson. 2002. Systematic data in biodiversity studies: use it or lose it. Systematic Biology 51: 303-316. DOI: https://doi.org/10.1080/10635150252899789

Gentry,A.H. 1995. Patterns of diversity and floristic composition in Neotropical montane forests, en S. Churchill, H. Balslev, E. Forero \& J. Luteyn (eds.), Biodiversity and conservation of Neotropical montane forests, pp. 103-126. Nueva York: The New York Botanical Garden.

Giraldo-Cañas, D. 1996. Malpighiaceae de Antioquia (Colombia): I. Diversidad, distribución geográfica y altitudinal y clave genérica basada en frutos. Universitas Scientiarum 3: 9-24.

Giraldo-Cañas, D. 2001. Relaciones fitogeográficas de las sierras y afloramientos rocosos de la Guayana colombiana: Un estudio preliminar. Revista Chilena de Historia Natural 74: 353-364. DOI: https://doi.org/10.4067/S0716078X2001000200012 
Giraldo-Cañas, D. 2008. Flora vascular de los afloramientos rocosos precámbricos (lajas-inselbergs) de la Amazonia colombiana y áreas adyacentes del Vichada: I. Composición y diversidad, en J. O. Rangel-Ch. (ed.), Colombia Diversidad Biótica VII: Vegetación, palinología y paleoecología de la Amazonia colombiana, pp. 89-118. Bogotá D. C.: Instituto de Ciencias Naturales, Universidad Nacional de Colombia.

Giraldo-Cañas, D. 2010. Distribución e invasión de gramíneas $\mathrm{C}_{3} \mathrm{y} \mathrm{C}_{4}$ (Poaceae) en un gradiente altitudinal de los Andes de Colombia. Caldasia 32: 65-86.

Giraldo-Cañas, D. 2011. Malpighiaceae, en Á. Idárraga Piedrahíta, R. Ortiz, R. Callejas Posada \& M. Merello (eds.), Flora de Antioquia. Catálogo de las plantas vasculares 2, pp. 571-577. Bogotá D. C.: Universidad de Antioquia, Missouri Botanical Garden, Oficina de Planeación de la Gobernación de Antioquia y Editorial D’Vinni.

Giraldo-Cañas, D. 2014. Riqueza y distribución altitudinal de gramíneas $\mathrm{C}_{3}$ y $\mathrm{C}_{4}$ en la Guayana venezolana. Ciencia en Desarrollo 5: 77-84. DOI: https://doi. org/10.19053/01217488.3233

Giraldo-Cañas, D. 2018. Circunscripción morfológica, diversidad, patrones de distribución y catálogo de la familia neotropical Marcgraviaceae (Ericales). Biota Colombiana 19: 49-69. DOI: https://doi.org/10.21068/c2018.v19n01a04

Gradstein, R. \& J. Uribe-M. 2011. A synopsis of the Frullaniaceae (Marchantiphyta) from Colombia. Caldasia 33: 347-376.

Grytnes, J. A. \& O. R. Vetaas. 2002. Species richness and altitude: a comparison between Null Models and interpolated plant species richness along the Himalayan altitudinal gradient, Nepal. American Naturalist 159: 294-304.

Hernández-Ruedas, M. A.; Y. Gómez-Ortiz, L. Herrera-Alsina, C. X. Pérez-Hernández. 2019. La diversidad filogenética y su utilidad para la conservación de la biodiversidad, en C. E. Moreno (ed.), La biodiversidad en un mundo cambiante: Fundamentos teóricos y metodológicos para su estudio, pp. 307-323. México D. F.: Universidad Autónoma del Estado de Hidalgo-Libermex.

Kessler, M. 2002. The elevational gradient of Andean plant endemism: varying influences of taxon-specific traits and topography at different taxonomic levels. Journal of Biogeography 29: 1159-1165. DOI: https://doi.org/10.1046/ j.1365-2699.2002.00773.x

Kessler, M. \& J. Kluge. 2008. Diversity and endemism in tropical montane forests from patterns to processes. Biodiversity and Ecology Series 2: 35-50.

Lægaard, S. 1999. Biogeography of Ecuadorian grasses. Arnaldoa 6: 185-196.

Linares, E. 2001. Aproximación al conocimiento de los bejucos de Colombia. Caldasia 23: 169-179.
Llopis-Belenguer, C.; I. Blasco-Costa \& J. A. Balbuena. 2018. Más allá del recuento de especies. Una nueva manera de enfocar la biodiversidad. Mètode Science Studies Journal 98: 47-51.

Lloret, F. \& J. M. González-Mancebo. 2011. Altitudinal distribution patterns of bryophytes in the Canary Islands and vulnerability to climate change. Flora 206: 769-781. DOI: https://doi.org/10.1016/j.flora.2011.04.007

Mamede, M. C. H.; A. M. Araujo Amorim \& R. Sebastiani. 2010. Malpighiaceae, en R. C. Forzza, J. F. A. Baumgratz, C. E. M. Bicudo, A. A. Carvalho Jr., A. Costa, D. P. Costa, M. Hopkins, P. M. Leitman, L. G. Lohmann, L. Costa Maia, G. Martinelli, M. Menezes, M. P. Morim, M. A. Nadruz Coelho, A. L. Peixoto, J. Rubens Pirani, J. Prado, L. P. Queiroz, V. C. Souza, J. Renato Stehmann, L. S. Sylvestre, B. M. T. Walter \& D. Zappi (eds.), Catálogo de plantas e fungos do Brasil 2, pp. 1183-1201. Rio de Janeiro: Instituto de Pesquisas Jardim Botânico do Rio de Janeiro.

Noguera-Urbano, E. A. 2017. El endemismo: diferenciación del término, métodos y aplicaciones. Acta Zoológica Mexicana (nueva serie) 33: 89-107.

Oommen, M. A. \& K. Shanker. 2005. Elevational species richness patterns emerge from multiple local mechanisms in Himalayan woody plants. Ecology 86: 3039-3047. DOI: https://doi.org/10.1890/04-1837

Prósperi, J.; G. Caballé \& Y. Caraglio. 2001. Lianas and hemiepiphytes: distribution, development, and adaptations. Selbyana 22: 197-212.

Rangel-Ch., J. O. 2006. La biodiversidad de Colombia. Palimpsesto 5: 292-304.

Rangel-Ch., J. O. 2015. La biodiversidad de Colombia: significado y distribución regional. Revista Academia Colombiana de Ciencias Exactas, Físicas y Naturales 39: 176-200. DOI: https://doi.org/10.18257/raccefyn.136

Stevens, G. C. 1992. The elevational gradient in altitudinal range: an extension of Rapoport's latitudinal rule to altitude. American Naturalist 140: 893-911.

Thiers, B. 2020 [continuously updated]. Index Herbariorum: A global directory of public herbaria and associated staff. New York Botanical Garden's Virtual Herbarium. Available from: https://sweetgum.nybg.org/ih/

Ulloa Ulloa, C.; P. Acevedo-Rodríguez, S. Beck, M. J. Belgrano, R. Bernal, P. E. Berry, L. Brako, M. Celis, G. Davidse, R. C. Forzza, S. R. Gradstein, O. Hokche, B. León, S. LeónYánez, R. E. Magill, D. A. Neill, M. Nee, P. H. Raven, H. Stimmel, M. T. Strong, J. L. Villaseñor, J. L. Zarucchi, F. O. Zuloaga \& P. M. Jørgensen. 2017. An integrated assessment of the vascular plant species of the Americas. Science 358: 1614-1617. DOI: https://doi.org/10.1126/science.aao0398

Villaseñor, J. L. 2016. Checklist of the native vascular plants of Mexico. Revista Mexicana de Biodiversidad 87: 559-902. 